\title{
Research on Emergency Resource Scheduling Decision-Making Based on Dynamic Information
}

\author{
Wang Guohua, He Yizhi, Cui Zhe* \\ School of Business, Nantong University, Nantong, China \\ Email address: \\ 7790@vip.163.com (Cui Zhe) \\ ${ }^{*}$ Corresponding author
}

To cite this article:

Wang Guohua, He Yizhi, Cui Zhe. Research on Emergency Resource Scheduling Decision-Making Based on Dynamic Information. Journal of Investment and Management. Vol. 6, No. 2, 2017, pp. 60-65. doi: 10.11648/j.jim.20170602.11

Received: April 7, 2017; Accepted: April 24, 2017; Published: June 7, 2017

\begin{abstract}
Emergency decision-making, especially emergency resource scheduling decision-making, is one of the key problems in emergency management. This article begins with the dynamism of emergency resources, provides a way of decision thinking about multi-stage scheduling of emergency resources based on the two-stage emergency rescue process and dynamic structure with continuous consumption, on the basis of the identification and integration of emergency resources.
\end{abstract}

Keywords: Emergency, Emergency Management, Emergency Decision-Making, Resource Scheduling

\section{Introduction}

In recent years, all kinds of public emergency events have always been under the spot light of the country and the society. With the deepening of understanding about emergency management, more and more countries and regions have put 'optimization of emergency allocation' on a significant strategic position to maximize the emergency rescue and guarantee functions of resources. However, under the current layout of all categories of emergency resources, how to recognize and integrate different kinds of emergency resources quickly and efficiently while facing emergencies with uncertainty and to form a scientific emergency rescue resource scheduling scheme has always been a tough problem. As is known to all, emergency management system is an open, complex and giant system, with attributes of multi-agent, multi-factor, multi-scale, multi-behavior and so on. Emergency decision-making under complex conditions, especially emergency resource scheduling decision-making, is one of the five key problems in the basic research on emergency management. This article begins with the dynamism of emergency resources, elaborates the identification and integration of emergency resources, and provides a way of decision thinking about multi-stage scheduling of emergency resources based on the two-stage emergency rescue process and dynamic structure with continuous consumption (shown in Figure 1).

\section{Identification of Emergency Resources with Dynamic Structure}

Broadly speaking, all resources that are needed in the process of emergency response can be called as emergency resources, that is to say, all the resources that are needed to guarantee the smooth response to emergency, and to maintain the normal production and living conditions of humans after the occurrence of a public emergency. These resources, however, whether they are natural or artificial, whether they are existing ones or potential ones, can all be categorized into emergency resources. However, due to the characteristics like suddenness, uncertainty, timeliness, periodicity, compulsoriness of emergency resources' demands, it's hard to estimate it accurately. So, people have to make quick changes to emergency resource scheduling scheme in accordance with the changes of emergency scenes and the proceeding of the emergency handling process. Because the differences in attributes like time, space, quantity, quality of emergency resources will lead to the presentation of multi-dimensional structure of these resources, the effective solution to this problem is to change the concepts and ways to integrate emergency resources, construct a system to identify 
emergency resources with dynamic structure and manage the resources. sources, demands, categories and classification of emergency

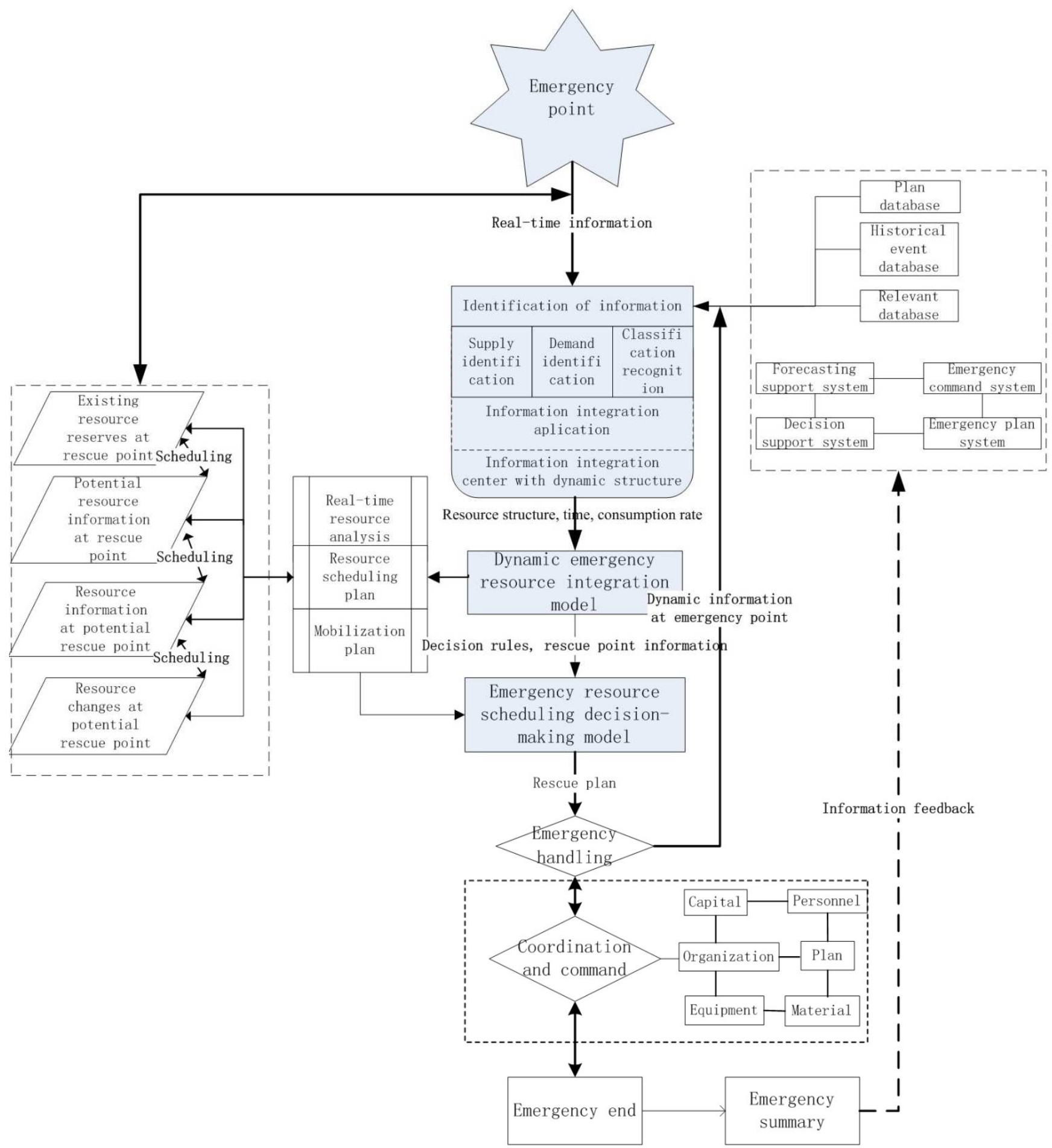

Figure 1. Emergency resource scheduling decision-making flowchart based on dynamic information.

(1) Source identification. Emergency resources can be allocated into two broad categories of existing resources and potential resources in accordance with their conditions. Existing resources include available resource reserves at each rescue point, normal outputs produced under the known production capacity, foreseen instant collections and so forth. Potential resources include all potential resource supplies, such as resources that are confiscated directly, produced by emergency production expansion, donated by other organizations or supplied spontaneously. Using contemporary information integration technology, emergency resources' attributes can be recognized and analyzed in the aspects of time, space and quantity. (1) Time dimension. It mainly means the time attributes of the process, of which comprises the 
mobilization, collection and emergency treatment of emergency resources in an emergency rescue. It includes: the generation characteristics, growth characteristics and rescuable conditions of resources. (2) Space dimension. It mainly means the distribution status and features of the geographical space at the rescue point. (3) Quantity dimension. It includes all kinds of existing resource reserves, all kinds of potential resource quantities and primary output of resources based on the maximum transportation capacity at one rescue point.

(2) Demand identification. Demand for emergency resources is the minimum resource demand when emergency is handled effectively. To handle emergency effectively, not only should the benefits outweigh the costs of handling, but also the resource should be used efficiently. Because of the characteristics like suddenness, uncertainty, timeliness, periodicity, compulsoriness of emergency resources' demand, the demand mix changes dynamically with the development of emergency and the implementation of emergency rescue. Demands for emergency resources can be described clearly by using the characteristics of resources in the aspects of their quantity, quality and structure.

(3) Classification identification. How to manage all kinds of emergency resources by categorizing and classifying them is of great significance to integrate and utilize emergency resources effectively and efficiently. Emergency resources are usually categorized by their application scope, urgency, use and demand drivers. Emergency resources' classification is determined dynamically in accordance with their own characteristics and the type, level and the characteristics of demand for emergency resources. One viable way of classifying emergency resources is to evaluate the indicators like timeliness, scarcity, substitutability and reliability of the resources, based on the identified source and demand, and to make decisions about classifying them by using relevant decision-making methods like evidential reasoning and fuzzy inference based on the integration of dynamic information, thus realizing the dynamic management of all kinds of emergency resources by classification and the guarantee of resources.

\section{Integration of Emergency Resources with Dynamic Structure}

In order to make sure that the emergency handling is not interrupted in an emergency rescue, the continuity of the consumption of emergency resources should be guaranteed. The instant consumption rate (consumed quantity of resources in unit time) of all kinds of emergency resources can be obtained in accordance with the characteristics of demand for resources at emergency point in an emergency, which stipulates the time at which the next batch of emergency resources must arrive. In addition, in order to schedule emergency resources effectively, it's necessary to dynamically integrate the layout of emergency resources at each rescue point, taking the consumption, scheduling time, velocity of all kinds of resources into account. When we have grasped the consumption rate of resources and generation characteristics of potential resources, resource reserves at each rescue point can be reduced effectively, resource supply schemes of each rescue point can be ascertained in accordance with existing resource reserves as well. In this way, focus can be put on demands as well as the process of how to satisfy them ultimately, thus replacing quantity with velocity through process optimization and improving the efficiency and effectiveness of emergency rescue at last.

The process of two-stage emergency rescue is shown below. In the first stage, after the occurrence of an emergency, relevant emergency resources should be transported to emergency points from a certain number of rescue points selected under the decision rules of minimum emergency response time and maximum transportation capacity of resources, in accordance with limited information grasped at emergency point. At the same time, the moment when the first batch of emergency resources arrives at the emergency point and is used up is marked as moment $t_{0}$ (which is also the moment when the second batch of emergency resources should arrive). In the second stage, the consumption rate of all kinds of emergency resources as well as the maximum transportation capacity constraint should be considered. It can be ascertained that the moment when the second batch of resources are used up is marked as $t_{1}$, based on the moment $t_{0}$ when the first batch of resources are used up. The moment when each batch of resources are used up afterwards is marked as $t_{2}, \ldots, t_{n}$. It needs to be assured that at any moment resources already arrived should satisfy the resource consumption in the emergency rescue, which means to guarantee the interval $\left(\Delta t_{i}{ }^{\prime}\right)$ between every two arrivals of resource scheduling is less than the consumption time of the last resource scheduling $\left(\Delta t_{i}=t_{i}-t_{i-1}\right)$. Obviously, the maximization of emergency resources' utilization is ensured. It should be noted that when $\Delta t_{i}$ is a constant value of $\Delta t$, the continuity and stability of emergency handling can be guaranteed. In order to ensure that, dynamic integration of every batch of transported emergency resources as well as that of rescue point should be used to balance the real time difference that emergency point causes upon each batch of resources' consumption. The integration of emergency resources should be carried out in the following aspects:

(1) Dynamic integration at rescue point. Dynamic integration at rescue point mainly ascertains the assemblage of alternative rescue points of each scheduling. Based on the two-stage process of emergency rescue, the first stage only involves one resource scheduling, while the second stage involves many times of resource scheduling. In the first stage, the information about emergency resources' conditions, the transportation capacity, the distance between rescue point and emergency point should be considered comprehensively to select relevant rescue point plan with the aim to achieve the minimum emergency response time and the maximum transportation capacity of resources. In the second stage, the selection of rescue point is mainly based on the rescue point in the first stage, the resource reserves at each rescue point, the 
consumption rate of all kinds of resources at emergency point, the resource reserves at potential rescue point, the transportation capacity at existing and potential rescue point, the distance between existing and potential rescue point and emergency point and such factors. The existing and potential rescue point should be ascertained dynamically in accordance with the three objectives of time (scheduling time), quantity (the quantity of emergency resources and structure) and cost (the number of rescue points and transportation capacity).

(2) Dynamic integration of resources at rescue point. The inconsistency between the real resource consumption time at emergency points is balanced mainly in the aspect of scheduling dynamic changes of the quantity of emergency resources, thus guaranteeing the consistency of the time $\Delta t$. Therefore, the core of research on integration of resources is the confirmation of the quantity of each kind of resources in each rescue at rescue point. The following things should be taken into consideration: (1) Resource reserves (residuals) at rescue point. To grasp the conditions of resource reserves at rescue point, not only the quantity of resource reserves at each rescue point and the structural characteristics of demands of emergency resources should be considered, but the resources at each rescue point should also be integrated in the three aspects of category, quantity and place. (2) Rescue resources at rescue point. The quantity of rescue resources should be ascertained in reference to factors like transportation capacity and transportation time, based on the comparison of demands of emergency resources and the resource reserves (residuals).

\section{Emergency Resource Scheduling Decision-Making with Multi-stage Dynamic Structure}

On the basis of identification and integration of emergency resources with dynamic structure, the construction of emergency resource scheduling decision-making model and mechanism is needed (shown in Figure 2). Emergency decision-making is multi-objective decision-making under complex circumstances. In the research of emergency decision-making model, the methods of operations research are applied extensively, including programming model, risk model, game model, markov model and so on. There are also some other researchers who introduce methods of group decision and decision simulation into the theoretical research of emergency decision-making. In the researches of existing literature, the common assumptions in the model are: There is only one emergency point (or potential emergency point), all the demands at emergency point are known and they are one-off (which means no new demands will occur), there are sufficient emergency resource reserves, all initial statuses in the system are known, and it only needs one calculation for the system to get the result.

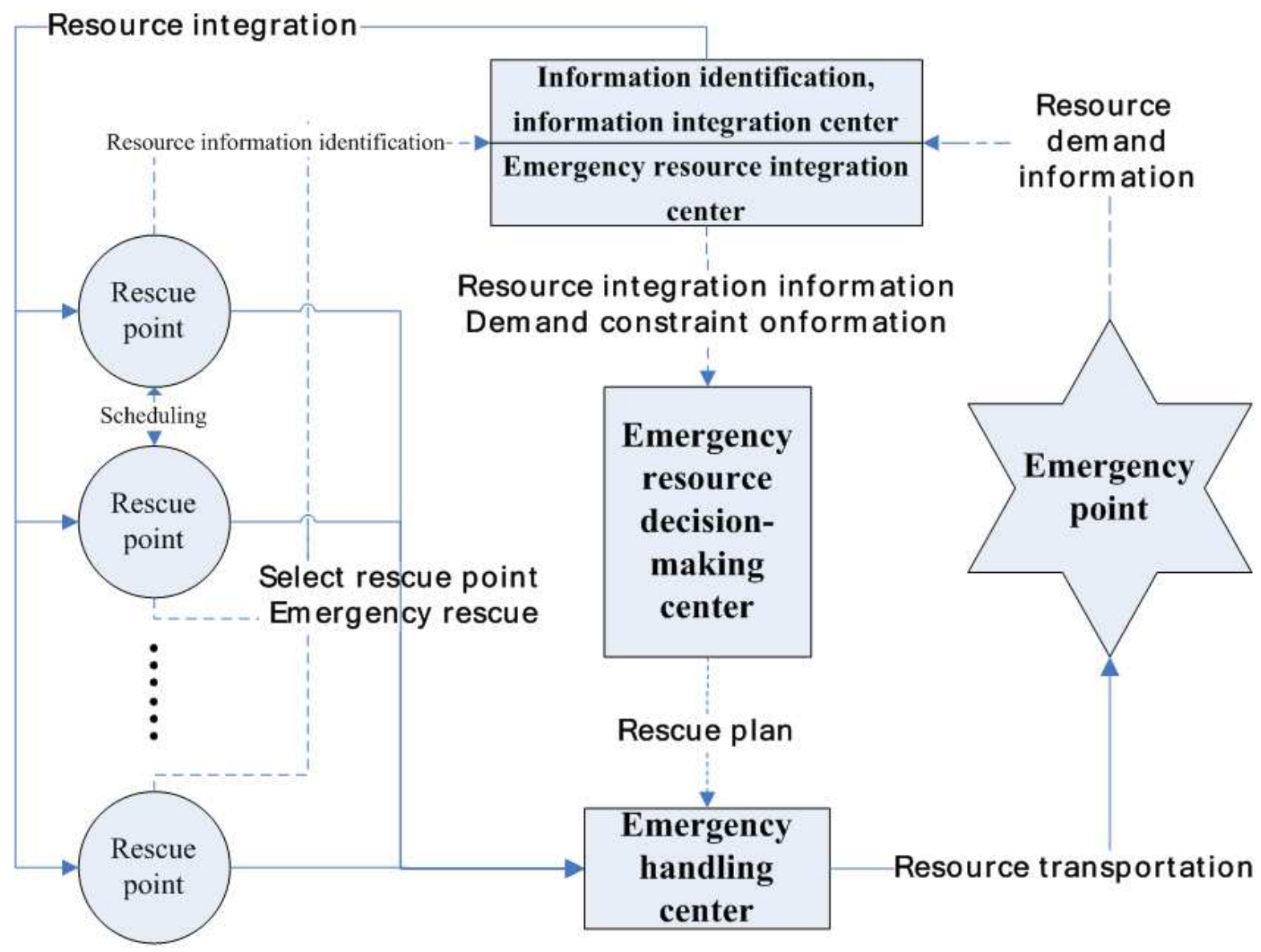

Figure 2. Emergency resource scheduling process with dynamic structure based on information integration and continuous consumption. 
In fact, because of the continuous dynamic change of the demand structure of emergency resources, the resource supplying quantity in one rescue point is limited, and the categories of resources can't be sure to fully satisfy emergency needs. In addition, in many circumstances, the instant transportation ability of emergency resources and the handling ability at emergency point after the occurrence of emergencies are limited, which can't satisfy the needs for one-off transportation. Therefore, to ensure the response to emergency begins as early as possible after emergency occurs, and to ensure that emergency resources with dynamic structure can satisfy continuous consumption conditions, it needs to phase and transport emergency resources continuously and dynamically and to ascertain the rescue point from aspects like transport time, quantity and structure of resources demanded, amount of rescue points and transport ability (shown in Figure $3)$. The main process includes:

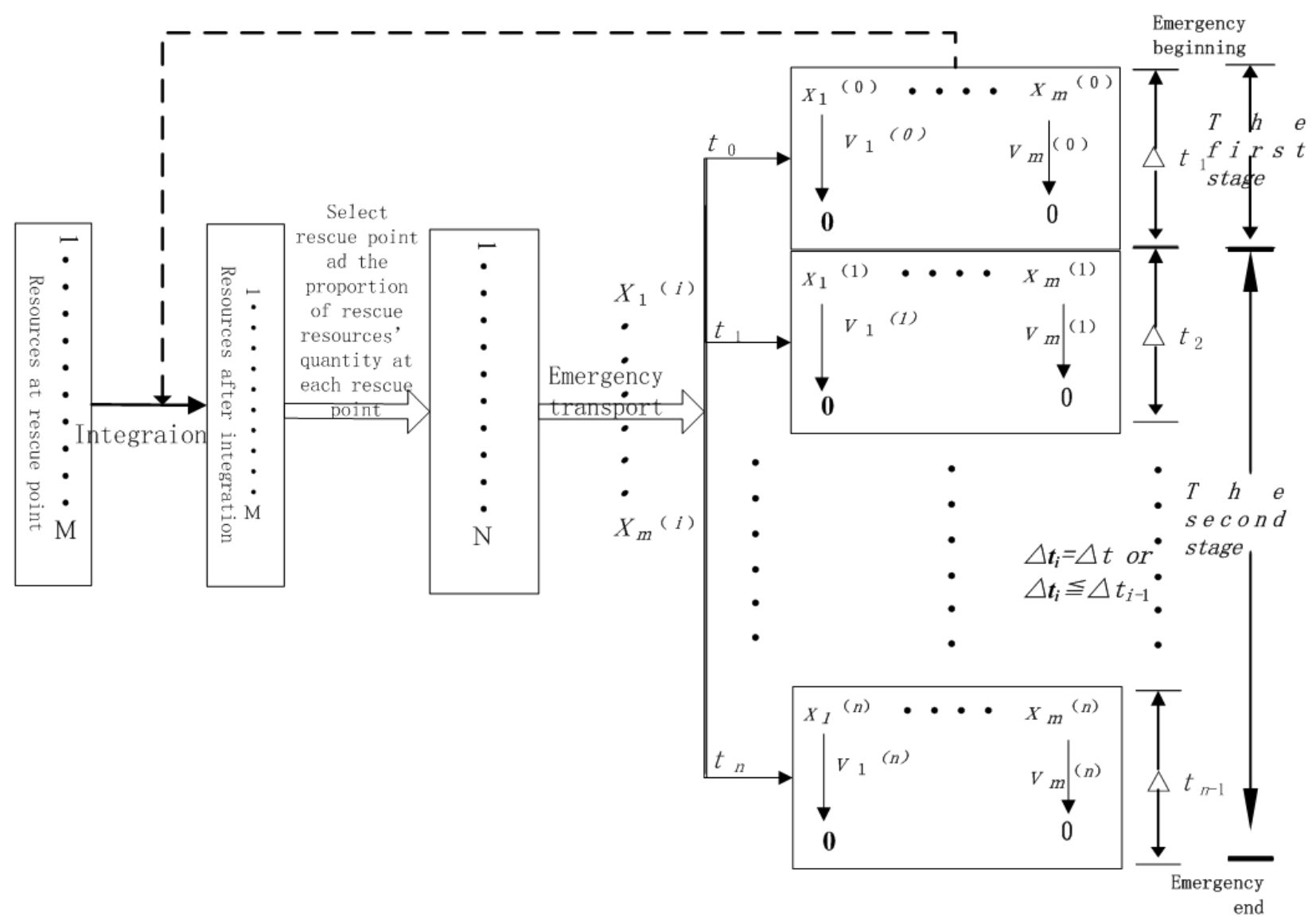

Figure 3. Two-stage decision-making of emergency resources' transportation.

(1) Multiple resource combination emergency scheduling based on emergency which begins the earliest. In order to initiate emergency response and the implementation process quickly after the occurrence of emergencies, it needs to transport the emergency resources which satisfy the structure of emergency demands to emergency points within the shortest time possible. The things needed to be considered here include the demand structure of emergency resources, the resource constraints at rescue point, the transport ability constraints and the selection of multiple rescue points. Based on the identification and integration of emergency resources, relations between structure and quantity of scheduling resources can be obtained, and the scheduling quantity of each kind of emergency resources can be arranged in accordance with the instant transport ability constraints, considering the characteristics and statuses of each kind of emergency resources. Theoretically, it can be guaranteed that each kind of emergency resources consumes the same time with emergency resource scheduling based on the consumption rate. Therefore, the problem of uncertainty about demand structure of various kinds of emergency resources can be solved.

(2) Multiple-stage emergency resource scheduling based on dynamic structure with continuous consumption. In order to make emergency resources with dynamic structure satisfy continuous consumption conditions, after the problem of earliest time of the beginning of emergency response being solved in the last step, this section mainly guarantees the quantity of resources already arrived can satisfy the resource consumption in the emergency rescue at any moment. No suspension problem of emergency activities shall occur because of the insufficient supply of resources, which means it should be guaranteed that the interval between of every two arrivals of resource scheduling is shorter than the consumption time of the last resource scheduling. Because the quantity of 
all kinds of emergency resources is limited at each emergency rescue point, resource conditions at each rescue point should be obtained first in accordance with the integration of emergency resources with dynamic structure in order to satisfy the compatibility between the demand structure and supply structure of emergency resources. Based on having obtained the information of alternative rescue points (constraints of resources' supply structure), the minimum time constraints of each emergency resource scheduling plan should be met for the continuous consumption of emergency resources.

(3) The optimization of multi-stage resource scheduling model. Here, the consistency of the consumption time of each kind of resources is guaranteed from the demand structure of each batch of resource scheduling, or say, it's assumed that the time is the same for a batch of resources to arrive at an emergency point from each rescue point. In practice, if a rescue plan with multiple rescue points is selected, the time point for a batch of resources to arrive at an emergency point from each rescue point is different as the distances and routes from each rescue point to the emergency point are different. The problem is actually the continuous problem of one-off scheduling model guaranteeing the consumption of resources. The problem can be solved perfectly through the refinement of the model or the optimization design of the emergency work flow. In the practice of emergency rescue, emergency handling also needs to consider the random destruction (transport efficiency) of resource scheduling routes, the capacity limitation, the resource handling ability of at emergency point and such aspects. In addition, improving the flexibility, reliability and applicability of emergency system is also an important issue to be considered.

\section{Conclusion}

Emergency management system is an open, complex and giant system, with attributes of multi-agent, multi-factor, multi-scale, multi-behavior and so on. This article begins with the dynamism of emergency resources, elaborates the identification and integration of emergency resources, and provides a way of decision thinking about multi-stage scheduling of emergency resources based on the two-stage emergency rescue process and dynamic structure with continuous consumption. It is hoped that this article can provide reference for the construction of emergency systems and the implementation of emergency decision-making.

\section{Acknowledgements}

Supported by National Natural Science Foundation of China (71101076);

Supported by the MOE Layout Foundation of Humanities and Social Sciences (10YJA630147);

Supported by Natural Science Foundation of Nantong University (10ZY002)

\section{References}

[1] Weicheng Fan. Thoughts and advice about the scientific problems in the emergency management of national public emergencies [J]. Science Foundation In China, 2007, (2): $71-76$

[2] Bo Shi, Jingguo Liang, Tuoliu. Researches on the resource allocation principles in public emergency management $[\mathrm{J}]$. Modern Management Science, 2008, (6): 88-89.

[3] Chunlin Liu, Jianmin He, Shanhan Sheng. Fuzzy programming methods of emergency system scheduling problems [J]. Journal of Systems Engineering, 1999, 14 (4): 351-355.

[4] Fiedrich F, Gehbauer F, RickersU. Optimized Resource Allocation for Emergency Response after Earthquake Disasters [J]. Safety Science, 2000, 35 (1): 41-57.

[5] Yingying $\mathrm{Yu}$, etc. Appraisal of resource distribution in emergency management and model, algorithm of model adjusted [J]. System Engineering, 2008, 26 (1): 75-81.

[6] Chunlin Liu, Jianmin He, Shanhan Sheng, Selection of the optimal plan in a multi-rescue point emergency system $[\mathrm{J}]$. Journal of Industrial Engineering and Engineering Management, 2000, 14 (1): 13-15.

[7] Shetty S R. An event driven single game solution for resource allocation in a multi-crisis environment [D]. Tampa; University South Florida, 2004.

[8] Wu Shihui, Yang Jianjun. Research on Dispatching Problem of Emergency System Resource for One - time Consumption [J]. Logistics Sci-tech, 2009 (7): 47-49.

[9] Wang Yu, He Jianmin. Research on Multi-resource Saving Scheme in Emergency System [J]. Journal of Southeast University (English Edition), 2002, 32 (3), 510-513.

[10] Zhiqing Zhang, Bao Xi, Zhonghua Yang, Xinxin Wang. Analysis of forecasts about supply chain coordination demands based on information fusion [J]. Science \& Technology Progress and Policy, 2008, 25 (12): 174-177. 